\title{
Adaptive Control of Multi-Rotor UAV Using Enhanced Learning Technology
}

\author{
Chin-Hsiung Lee \\ College of Robotics, Fuzhou Polytechnic, China
}

\begin{abstract}
Due to the development and maturation of embedded processor, micro-sensor technology and control theory, multi-rotor UAV is booming. The continuously increasing efficiency and multifunctionality make multi-rotor UAV widely used in military, civil aerial shooting, search and rescue, monitoring and other fields. With the special mechanical structure and dynamic characteristics, it also plays an important role in the scientific research. However, UAV is susceptible to the disturbance of environmental factors during the flight. This study uses reinforcement learning to enhance the stability of flight control of multi-rotor UAV. The reinforcement learning method, also known as reinforcement learning, is one of the learning methods in the field of machine learning and artificial intelligence. It is the most commonly used algorithm in the agent system, which is suitable for the unknown environment. In particular, the agent system will get feedback from changes in the environment, so it can learn from experience and optimize the overall benefit. This research mainly uses the Q-learning algorithm to realize the enhanced learning mechanism, which learns the best response and control corresponding to different UAV attitude, adopts the PID control parameter of reinforcement learning adjustment, and uses it to maintain the stable posture of UAV in the unknown environment.
\end{abstract}

Keywords-multi-rotor UAV; enhanced learning; PID control; machine learning; self-adaptive control

\section{INTRODUCTION}

In recent years, drones have developed rapidly. Whether from the aspect of application, enterprise management or domestic research field, the multi-rotor UAV has great development potential. Multi-rotor drones have unique advantages in a growing number of areas, such as high-risk electrical tower inspections, military reconnaissance, disaster monitoring, aerial photography, oil pipeline patrols, atmospheric sampling, traffic monitoring and support for largescale activities. Although the multi-rotor UAV is widely used, the flight stability of the UAV is easily affected by the environment, and its flight self-stable performance is weak and cannot be controlled from the remote control. The problem derived from the stability of multi-rotor UAV is a great hindrance to the research of its applications. Therefore, this research is devoted to the study of a stable flight control system which can realize self-stabilization and autonomous flight to meet the needs of UAV operation.

The PID controller is a co mmon feedback mechanism in the control application, and its technology mainly cooperate with the motor encoder and the adjustment controller in proportion, integral, differential parameters, to solve the problem of multi-rotor UAV control. While the development of machine learning technology is quite extensive in various fields, how to apply it to the balance and stability of multi-rotor UAV has become the main research target. This study aims to solve the problem of balancing stability control of multi-rotor UAV, which can make the UAV self-balancing by machine learning. When the control instruction is given to the multi-rotor UAV, the PID controller ensures that the multi-rotor UAV will make corresponding action according to the instructions received. The multi-rotor UAV can be used in a variety of environments to complete different specified tasks. It can also combine the indoor localization, roadblock detection, path plan and many more other techniques, to make the control instruction more elastic and optimal. Through the self-training of enhanced learning, the unmanned function of multi-rotor is selfstabilizing and capable of autonomous flying.

\section{DYNAMIC MODEL OF FOUR-ROTOR UAV}

Four-rotor UAV has been widely used in various fields. It consists of a fuselage and four rotors. The rotors are divided into two opposing groups, to counteract the torsional torque produced by rotation. Rolling and pitching can be achieved by changing the rotational speed of the rotor in the same group by differential motion. Yawing can be achieved by controlling the torsional torque of two sets of rotors. Since the four-rotor UAV has only four driving forces, it needs to complete six degrees of freedom movement. The modeling assumptions are as follows:

- The four-rotor UAV is a uniformly symmetrical rigid structure.

- The body's center of gravity coincides with the body's coordinate origin.

- The propellers are rigid.

- The lift of the four-rotor UAV is proportional to the square of the propeller speed.

As shown in Figure I, the two relative coordinates are the inertial coordinate $E\left(x_{e}, y_{e}, z_{e}\right)$ and the body coordinate $B\left(x_{b}, y_{b}, z_{b}\right)$ respectively. By aviation practice, the absolute position of the four-rotor drone is $X=\left[\begin{array}{lll}x & y & z\end{array}\right]^{T}$, and the attitude Euler angle is $\Theta=\left[\begin{array}{lll}\phi & \theta & \varphi\end{array}\right]^{T}$. Where $\phi$ is the roll angle, $\theta$ is the pitch angle, and $\varphi$ is the yaw angle. Assuming that the angular rate of body is $\Omega=\left[\begin{array}{lll}\omega_{x b} & \omega_{y b} & \omega_{z b}\end{array}\right]^{T}$, then: 


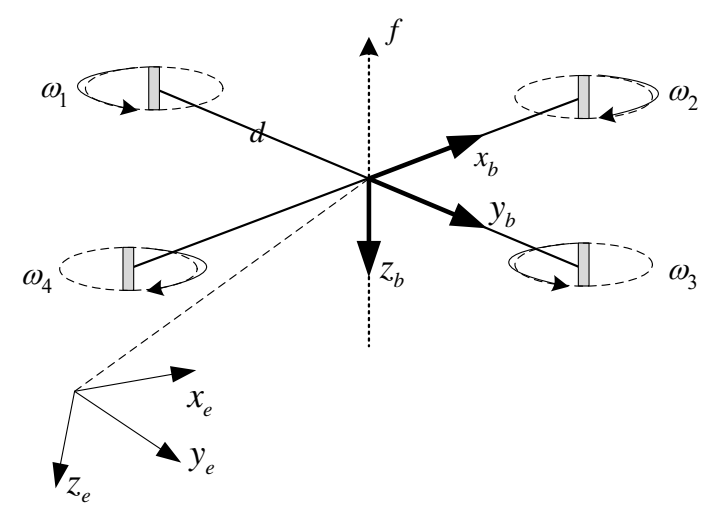

FIGURE I. STRUCTURAL MODEL OF FOUR-ROTOR UAV

$$
\left[\begin{array}{c}
\omega_{x b} \\
\omega_{y b} \\
\omega_{z b}
\end{array}\right]=\left[\begin{array}{ccc}
1 & 0 & -\sin \theta \\
0 & \cos \phi & \cos \theta \sin \phi \\
0 & -\sin \phi & \cos \theta \cos \phi
\end{array}\right]\left[\begin{array}{c}
\dot{\phi} \\
\dot{\theta} \\
\dot{\varphi}
\end{array}\right]
$$

The total pulling force acting on the body is

$$
f=C_{T}\left(\omega_{1}^{2}+\omega_{2}^{2}+\omega_{3}^{2}+\omega_{4}^{2}\right)
$$

For the X-type 4-rotor drone, the torque generated by the rotor is

$$
\left[\begin{array}{c}
f \\
\tau_{x} \\
\tau_{y} \\
\tau_{z}
\end{array}\right]=\left[\begin{array}{cccc}
C_{T} & C_{T} & C_{T} & C_{T} \\
\frac{1}{\sqrt{2}} d C_{T} & -\frac{1}{\sqrt{2}} d C_{T} & -\frac{1}{\sqrt{2}} d C_{T} & \frac{1}{\sqrt{2}} d C_{T} \\
\frac{1}{\sqrt{2}} d C_{T} & \frac{1}{\sqrt{2}} d C_{T} & -\frac{1}{\sqrt{2}} d C_{T} & -\frac{1}{\sqrt{2}} d C_{T} \\
C_{M} & -C_{M} & C_{M} & -C_{M}
\end{array}\right]\left[\begin{array}{c}
\omega_{1}^{2} \\
\omega_{2}^{2} \\
\omega_{3}^{2} \\
\omega_{3}^{2}
\end{array}\right]
$$

As shown in Figure II, the four-rotor UAV system has 4 inputs (total pulling force $f$ and three axis torques $\tau$ ) and controls 6 outputs (position $p_{e}$ and attitude angle $\Theta$ ). The linear simplified model of 4 rotor UAV is

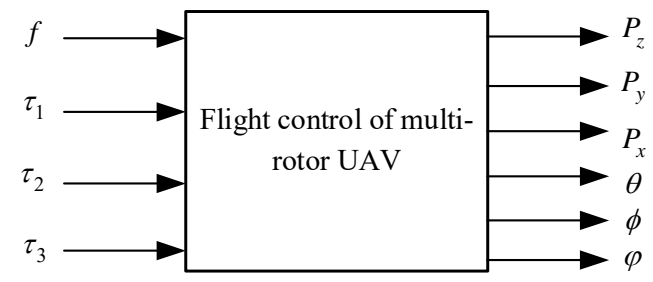

FIGURE II. INPUTS AND OUTPUTS OF MULTI-ROTOR UAV

$$
\begin{aligned}
& \dot{p}_{e}=v_{e} \\
& \dot{v}_{e}=g e_{3}-\frac{f}{m} R_{b}^{e} \cdot e_{3} \\
& \dot{\Theta}=W \Omega \\
& J \dot{\Omega}=\tau
\end{aligned}
$$

\section{PID CONTROL AND MACHINE LEARNING}

PID control is one of the earliest control methods. Because of its simple algorithm and high reliability, it is widely used in industrial process control. It is also very suitable for drone control. The differential equation of the PID controller is

$$
u(t)=k_{p} e(t)+k_{i} \int_{0}^{t} e(\tau) d \tau+k_{d} \frac{d e(t)}{d t}
$$

The PID controller's proportional parameter P, the integral parameter I, and the differential parameter D respectively correspond to the current error, the past accumulative error and the future error. By adjusting the PID controller three parameters can adjust the control system, and meet the requirements. A typical single-stage PID controller is shown in Figure III. However, since the PID parameters are fixed, they will not change with the environment. Machine learning is a very good choice in order to enable UAVs to resist external interference factors.

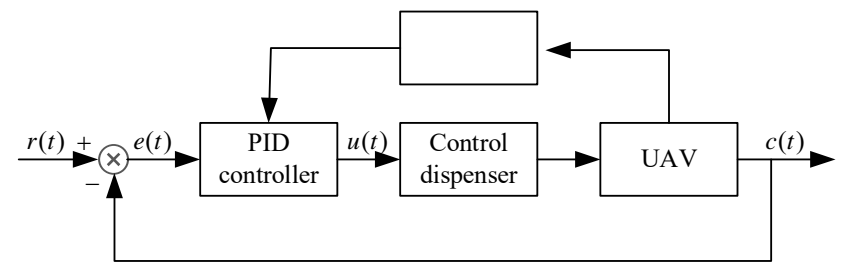

FIGURE III. TYPICAL PID CONTROLLER WITH MACHINE LEARNING STRUCTURE DIAGRAM.

Reinforcement learning is a machine learning algorithm that emphasizes how to take action based on the environment to achieve maximum expected benefit. Q-learning belongs to the classical algorithm of reinforcement learning, which is used to solve Markov decision problem. Which depending on the state observed at each moment, an action is chosen from the available set of actions to make a decision and the next (future) state of the system.

The Markov decision process can be described as: a UAV (agent) changes its state by acting (action), and interacts with the environment (E) to be rewarded (reward). The goal is to get the best results through a certain action strategy $(\pi)$. It is composed of 5 elements:

S: A set of all possible states.

A: Action that can be made under state $\mathrm{S}$.

$\mathrm{P}: \mathrm{P}_{\mathrm{A}}\left(\mathrm{S}, \mathrm{S}^{\prime}\right)$, which represents the probability of from state $\mathrm{S}(\mathrm{t})$ conversion to state $\mathrm{S}^{\prime}(\mathrm{t}+1)$ under action $\mathrm{A}$. 


\section{$\gamma$ : Attenuation variable.}

$\mathrm{V}$ : The value of the measurement strategy $(\pi)$ is related to the current immediate return and future expected return.

$$
\begin{aligned}
& V(S)=E[U \mid S(t)] \\
& U=R(t+1)+\gamma \cdot R(t+2)+\gamma^{2} \cdot R(t+3)+\ldots
\end{aligned}
$$

Where $R(t+n)$ indicates the return at the $t+n$ moment.

\section{SimUlation RESUltS}

To verify the effectiveness of the design method, we have carried out a series of Matlab-based simulations. Taking the model (4) of the four-rotor UAV as the plant, the entire flight control system is tested in the simulation. The parameters of a UAV dynamic model are

$$
m=0.5 \mathrm{~kg}, g=9.8 \mathrm{~m} / \mathrm{s}^{2}, d=225 \mathrm{~mm}
$$

At first, the related PID controller parameters are chosen as

$$
K_{p}=10, K_{i}=10, K_{d}=1
$$

However, the time responses of an unadjusted PID control is unsatisfactory. In order to achieve better transient state and steady-state performance, we used the enhanced learning method to improve PID control. Adjust PID parameters at any time due to feedback and environmental changes. The results of Matlab/simulink simulation show that the four-rotor UAV has good performance in stability and transient responses under PID control with reinforcement learning. The time responses of the PID control vs PID control with machine learning are shown in Figure IV. Where $X=\left[\begin{array}{lll}x & y & z\end{array}\right]^{T}$ is the output state of a typical PID control, and $X^{\prime}=\left[\begin{array}{lll}x^{\prime} & y^{\prime} & z^{\prime}\end{array}\right]^{T}$ is the output state of the PID control with machine learning. After 0.5 seconds, the PID parameters adjusted by machine learning are

$$
K_{p}=25, K_{i}=15, K_{d}=8
$$

Illustrated by the simulation, the method of machine learning is applied to adjust the flight control parameters of four-rotor UAV and to optimize the parameters. This control mode also has the capability of anti-disturbance and rapid reaction.
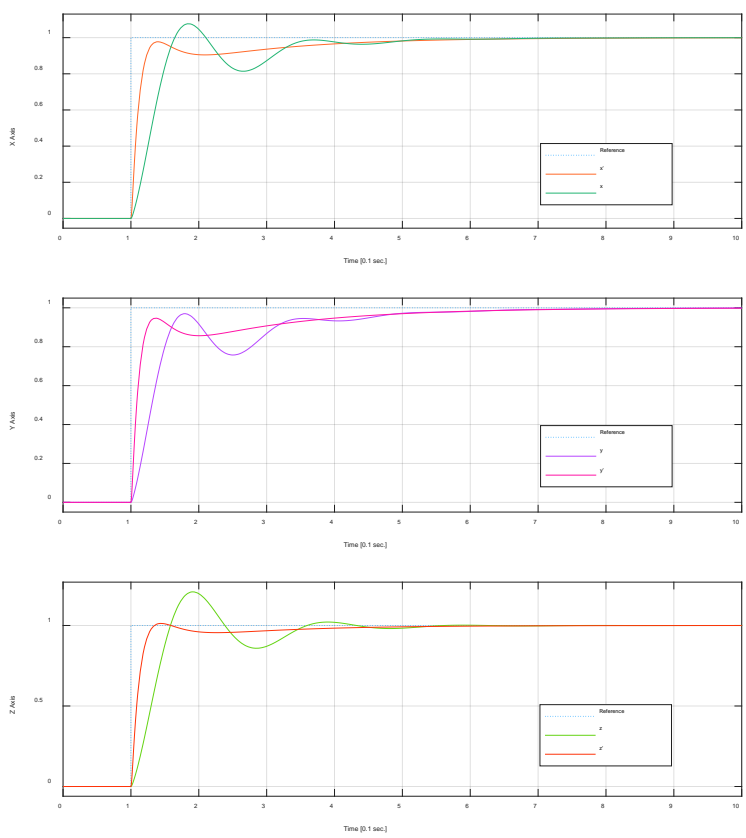

FIGURE IV. TIME RESPONSES OF THE PID CONTROL VS PID CONTROL WITH MACHINE LEARNING

\section{CONCLUSIONS}

This paper presents a PID control algorithm based on machine learning optimization for multi-rotor UAV, and continued with the simulation and experimental research of the calculator. The results show that the PID controller based on machine learning optimization design is significantly more robust. The control performance of the system still has good dynamic quality and steady state accuracy even under the condition of the external environment. Machine learning allows drones to improve algorithms through experience or data. Being able to learn from a large amount of data, the algorithms allow drones to achieve a pattern and use this model to predict future optimization of flight control parameters.

\section{ACKNOWLEDGMENT}

This work was supported by Scientific Research Startup Foundation of Fuzhou Polytechnic under project RCQD201802.

\section{REFERENCES}

[1] Stephen P. Tseng, Wen-Lung Li, Chih-Yang Sheng, Jia-Wei Hsu, ChinSheng Chen, "Motion and attitude estimation using inertial measurements with complementary filter," Control Conference (ASCC), May 2011

[2] S. Laliberte and A. Rango, "Texture and scale in object-based analysis of sub-decimeter resolution unmanned aerial vehicle (UAV) imagery," IEEE Trans. Geosci. Rem. Sens. 47, 761-770, 2009.

[3] P. Murrieri S. Bouabdallah and R. Siegwart. Design and control of an indoor micro quadrotor. Porceedings of the 2004 IEEE International Conference on Robotics \& Automation, April 2004.

[4] Yi-Hua Lin, The Autonomous Position Flight Control For a Quadrotor, TaTung University Department of Electrical Engineering, 2013.

[5] Yi-Jhan Dai, Bao-Chiang Hwang, Bih-Sheng Hsu, Balance Control for Quadrotor Helicopter, Bulletin of the College of Engineering National Ilan University No. 8, P. 152-170, December 2012. 
[6] Mark Euston, Paul Coote, Robert Mahony, Jonghyuk Kim, and Tarek Hamel. A co mplementary filter for attitude estimation of a fixed-wing uav with a low-cost imu. In 6th International Conference on Field and Service Robotics, July 2007.

[7] Kallapur, I. Petersen, and S. Anavatti. A robust gyroless attitude estimation scheme for a s mall fixed-wing unmanned aerial vehicle. pages 666-671, aug. 2009.

[8] Xiaogang Ruan, Jianxian Cai, Jing Chen, "Learning to Control TwoWheeled SelfBalancing Robot Using Reinforcement Learning Rules and Fuzzy Neural Networks," Natural Computation, 2008. ICNC '08. Fourth International Conference on, Oct. 2008, pp. $395-398$

[9] Bouabdallah, S. Design and control of quadrotors with application to autonomous flying, EPFL. 2007

[10] Beyeler, J.-C. Zufferey, and D. Floreano. Vision-based control of nearobstacle flight. Autonomous Robots, 27:201-219, 2009.

[11] J.-P. Bristeau, F. Callou, D. Vissiere, and N. Petit. The navigation and control technology inside the ar.drone micro uav. In 18th IFAC World Congress, pages 1477-1484, Milano, Italy, 2011.

[12] F. Fraundorfer, L. Heng, D. Honegger, G. Lee, L. Meier, P. Tanskanen, and M. Pollefeys. Vision-Based Autonomous Mapping and Exploration Using a Quadrotor MAV. In Intelligent Robots and Systems (IROS), 2012 IEEE/RSJ International Conference on, Oct 2012.

[13] Farid Kendoul, Isabelle Fantoni, and Kenzo Nonami. Optic flow-based vision system for autonomous $3 \mathrm{~d}$ localization and control of small aerial vehicles. Robotics and Autonomous Systems, 57(67):591 - 602, 2009.

[14] S. Zingg, D. Scaramuzza, S. Weiss, and R. Siegwart. MAV navigation through indoor corridors using optical flow. In Robotics and Automation (ICRA), 2010 IEEE International Conference on, pages $3361-3368$, may 2010.

[15] J.-C. Zufferey, A. Beyeler, and D. Floreano. Autonomous flight at low altitude using light sensors and little computational power. International Journal of Micro Air Vehicles, 2:107-117, 2010.

[16] Lim, H., et al. "Build Your Own Quadrotor: Open-Source Projects on Unmanned Aerial Vehicles." IEEE Robotics \& Automation Magazine 19(3): 33-45, 2012.

[17] Leishman, R. C., et al. "Quadrotors and Accelerometers: State Estimation with an Improved Dynamic Model." IEEE Control Systems 34(1): 28-41, 2014.

[18] Grzonka, S., et al. "A Fully Autonomous Indoor Quadrotor." IEEE Transactions on Robotics 28(1): 90-100, 2012.

[19] Alexis, K., et al. "Model predictive quadrotor control: attitude, altitude and position experimental studies." IET Control Theory \& Applications 6(12): 1812-1827, 2012.

[20] Raffo, G. V., et al. An underactuated $\mathrm{H} \infty$ control strategy for a quadrotor helicopter. Control Conference (ECC), 2009 European.

[21] Yang Rongfeng, four-rotor attitude control, National Cheng Kung University, Aviation Space Engineering master thesis.

[22] Yao Lei, Biogeography, stability control of four-rotor UAV, master thesis of Electrical Engineering, National Taipei University, Science and Technology master thesis.

[23] Wang Wen-jian, Yuan Liang, "Attitude Control of Four-Rotor Aircraft Via Improved Fuzzy PID." Machinery Design \& Manufacture, vol.8: pp. 9-12, 2017.

[24] Xu Jing, Cai Chen-xian, Li Yong-qi, Zou Yun, "Dual-loop path tracking and control for quad-rotor minature unmanned aerial vehicles." Control Theory \& Applications, 32(10): pp.1335-1342, 2015. 